\title{
BMJ Open Healthy Ageing and Biomarkers Cohort Study (HABCS): a cohort profile
}

\author{
Yuebin Lv, ${ }^{1}$ Chen Mao, ${ }^{2}$ Zhaoxue Yin, ${ }^{3}$ Furong Li, ${ }^{2}$ Xianbo Wu, ${ }^{2}$ Xiaoming Shi ${ }^{1}$
}

To cite: Lv Y, Mao C, Yin Z, et al. Healthy Ageing and Biomarkers Cohort Study (HABCS): a cohort profile. BMJ Open 2019;9:e026513. doi:10.1136/ bmjopen-2018-026513

- Prepublication history for this paper is available online. To view these files, please visit the journal online (http://dx.doi org/10.1136/bmjopen-2018026513)

YL and CM contributed equally.

Received 07 September 2018 Revised 02 April 2019 Accepted 19 August 2019

Check for updates

(C) Author(s) (or their employer(s)) 2019. Re-use permitted under CC BY-NC. No commercial re-use. See rights and permissions. Published by BMJ.

${ }^{1}$ National Institute of Environmental Health, Chinese Center for Disease Control and Prevention, Beijing, China ${ }^{2}$ Department of Epidemiology, School of Public Health, Southern Medical University, Guangzhou, China

${ }^{3}$ Division of Non-Communicable Disease Control and Community Health, Chinese Center for Disease Control and Prevention, Beijing, China

Correspondence to

Dr Xiaoming Shi;

shixm@chinacdc.cn

\section{ABSTRACT}

Purpose The Chinese Longitudinal Healthy Longevity Survey Biomarkers Cohort (Healthy Ageing and Biomarkers Cohort Study (HABCS)) was established to investigate the determinants of healthy aging and mortality among the oldest old in China. Besides collecting health status, behavioural and sociodemographic circumstances, the present study also gathers comprehensive data for the elderly by simultaneously collecting, detecting, analysing blood and urine, respectively.

Participants HABCS is a community-based longitudinal multiwave study of older men and women aged 65 or above. Baseline survey and the follow-up surveys with replacement for deceased elderly were conducted in eight longevity areas in China, which cover the northern, middle and southern parts of China. Between 2008 and 2017, 6333 participants were included in HABCS, comprising 1385 centenarians, 1350 nonagenarians, 1294 octogenarians, 1577 younger elderly (aged 65-79). Findings to date We have found that higher baseline levels of (1) total cholesterol, (2) low-density lipoprotein cholesterol (LDL-C) and (3) superoxide dismutase activity were associated with greater cognitive decline. While (4) higher LDL-C level was associated with lower risk of allcause mortality. There was a reverse association between (5) plasma vitamin D and cognitive impairment in crosssectional and prospective study.

Future plans We are currently exploring the relationships between various biomarkers and different outcomes such as cognitive function and mortality. This longitudinal cohort study will be continued in the future.

\section{INTRODUCTION}

Population aging is one of the major challenges facing most countries in the world due to the declines in fertility and increases in life expectancy. ${ }^{1}$ As for China, since the stringent implementation of population and family planning policies in the last three decades, the pace of population ageing is even more rapid than other countries. According to the 2015 census, population aged 60 and over is 222 million in China, which accounts for $16 \%$ of its total population, and by 2050 this is projected to reach 400 million or more, of which the oldest old (individuals older than 80 years) will reach 150 million. As the most rapidly growing portion of the older population, ${ }^{2}$ the oldest old is presenting a major challenge for
Strengths and limitations of this study

- We select a representative sample of centenarians, nonagenarians, octogenarians and younger elderly from eight longevity areas in China, which may offer a unique data set of oldest old in a non-Western country.

- The Healthy Aging and Biomarkers Cohort Study provides data on under-represented age groups (eg, centenarians), for whom comparisons often lack statistical power due to limited sample sizes in most other studies.

- All the interviewees are interviewed face to face at home, thus limiting the under-representation of frailer participants.

- This study mainly focuses on Chinese oldest old, so the findings may not be applicable to other ethnic or age groups.

Due to the special sampling design, the study population may not be a truly random sample representative of the entire population.

health and social care systems because they often require much more daily assistance and medical care. ${ }^{3}$ Thus, it is strategically important to gain a better understanding of the determinants of healthy aging and mortality in the oldest old so to be able to reduce the negative impacts of rapid aging.

Chinese Longitudinal Healthy Longevity Survey (CLHLS) was established in China since 1998, as a sister study to other elderly cohorts (eg, the Health and Retirement Study (HRS) in the USA, the Survey of Health, Ageing and Retirement (SHARE) in Europe, and Longitudinal Aging Study in India). The CLHLS is a community-based longitudinal cohort with the aim of examining factors that promote longevity and quality of life among the older adults. However, we have noticed that the CLHLS study has been limited principally to social science research, ${ }^{3-18}$ whereas other objective medical aspects such as biomarkers are not available. As a result, such a major obstacle has been preventing us from developing a complete understanding of the determinants of healthy aging. Therefore, in order to address this weakness, we established 


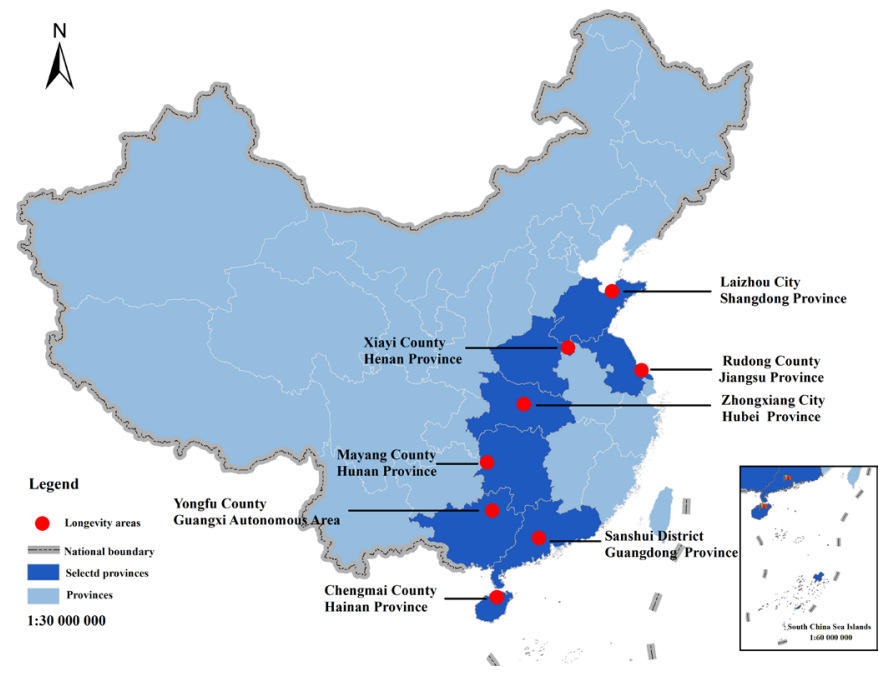

Figure 1 Selected longevity areas in China for sampling in HABCS. HABCS, Healthy Aging and Biomarkers Cohort Study.

a subcohort (The Healthy Ageing and Biomarkers Cohort Study (HABCS) ) in eight longevity areas from CLHLS in China in 2008. The HABCS covers similar domains that CLHLS has investigated, and provides more sophisticated data for the oldest old by simultaneously collecting, detecting, analysing blood and urine, respectively. As a result, the HABCS complements the epidemiological profile of CLHLS by examining objective biological indicators, which would provide more practical implications for the policy of healthcare in an ageing society.

\section{COHORT DESCRIPTION}

\section{Participants of HABCS}

HABCS is a community-based longitudinal multiwave study of older people aged 65 or above. The baseline survey and the follow-up surveys with replacement for deceased elderly are conducted in eight longevity areas with exceptionally high densities of centenarians. These longevity areas were selected by the Chinese Society of Gerontology, which cover the northern, middle and southern parts of China. They are Laizhou City of Shandong Province, Xiayi County of Henan Province, Zhongxiang City of Hubei Province, Mayang County of Hunan Province, Yongfu County of Guangxi Autonomous Area, Sanshui District of Guangdong Province, Chengmai County of Hainan Province and Rudong County of Jiangsu Province ${ }^{19}$ (figure 1 ). We interview all

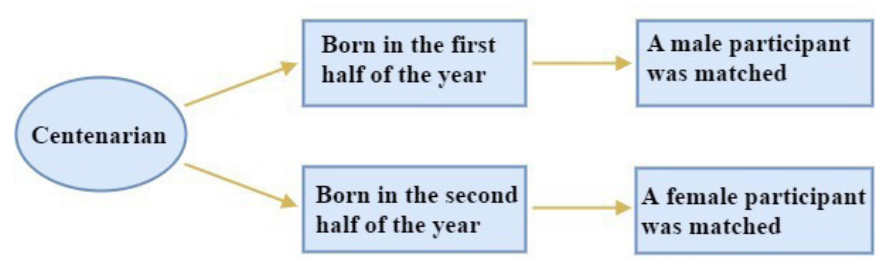

Figure 2 Matched-recruitment procedure of gender for a centenarian. centenarians who voluntarily agree to participate in the baseline survey and subsequent follow-up surveys face to face at home, aiming to keep a large subsample of centenarians in the sampled longevity areas. Interviewee's age was subject to careful validation. Investigators do not ask for age directly, but verify this variable by date of birth. Further, all reported dates of birth are validated by reference to the following information: household booklet, ID card, Chinese calendar birth date and animal year, genealogical records if available, children's ages, siblings' ages ${ }^{20}$ As far as possible, for each centenarian interviewee, one nearby octogenarian, one nearby nonagenarian and one older adults aged 70-79 years old of predefined age and sex are randomly interviewed. Specifically, if the number mantissa of centenarians in the very province is between 0 and 4 , one nonagenarian aged 90-94, one octogenarian aged $80-84$ and one younger elderly aged $70-74$ are interviewed nearby; on the other hand, if the number mantissa of centenarians is between 5 and 9 , one nonagenarian aged 95-99, one octogenarian aged 85-89 and one younger elderly aged 75-79 are interviewed nearby. In addition, 0.5 nearby elderly aged 65-69 of predefined age and sex are also randomly interviewed. 'Nearby' is loosely defined as in the same village or street, if available, or in the same town, same county or city. ${ }^{21}$ As for the gender matching, if a centenarian was born in the first half of the year, corresponding matched male participants will be selected randomly. Otherwise, female participants will be selected instead (figure 2). In general, women had a larger proportion in comparison to men among the oldest old, thus, we purposely try to have approximately equal numbers of male and female octogenarians and nonagenarians according to the frequency of the matched centenarians who reside nearby, and their age and sex are predesigned based on the centenarians' code numbers (randomly assigned) and birth month.

Those interviewees who are still surviving in the follow-up waves are reinterviewed, whereas those who are interviewed but subsequently die before the next wave will be replaced by new interviewees of the same sex and age (or within the same 5-year age group). We also try to reinterview those who lose contact previously but are still alive. All newly added interviewees have no kinship or family relationship with other participants. ${ }^{20}$

\section{Cohort follow-up and quality control}

We included seven longevity areas for baseline survey in 2008-2009 except for Rudong County (Jiangsu Province), which was added to the survey in 2012. Follow-up surveys were carried out in 2012, 2014 and 2017 to date. In all four waves, in total, 1385, 1350, 1294 and 1577 faceto-face interviews were conducted with centenarians, nonagenarians, octogenarians, younger elderly (aged 65-79), respectively (tables 1 and 2 show the detailed information). 
Table 2 Baseline characteristics of HABCS participants

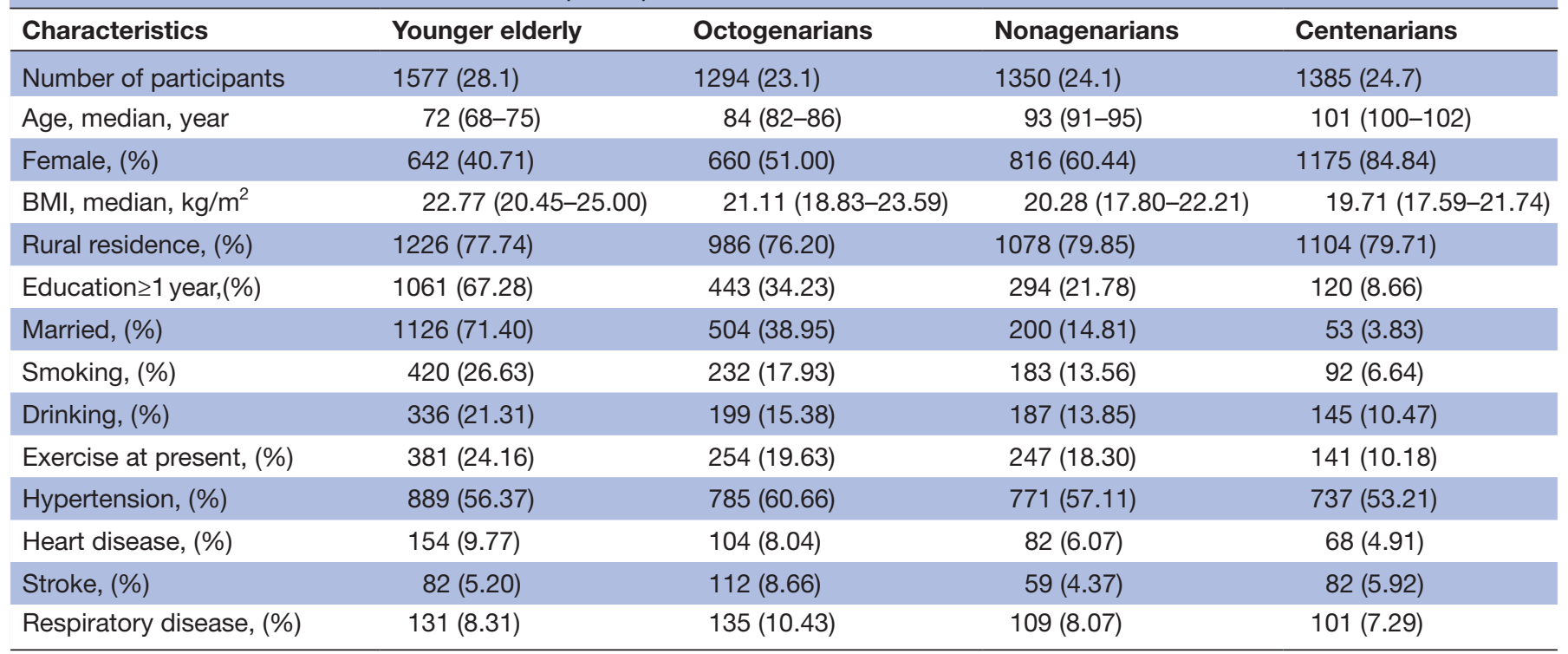

Continuous variables are expressed as medians and IQRs; categorical variables were expressed as frequencies (percentages). BMI, body mass index; CVD, cardiovascular disease; HABCS, Healthy Aging and Biomarkers Cohort Study.

The Chinese Center for Disease Control and Prevention (CCDC) performs overall project review and validation. The provincial Disease Control and Prevention (PCDC) provides technical guidance and participate in field quality control. Health clinics in towns and townships, community health service centres and village medical personnel also involve in the research work. Fieldwork is carried out by investigators composing of three members, including one group leader (responsible for work organisation and quality control), one interviewer (in charge of interview and filling out the questionnaires) and one doctor (responsible for health examination, collecting biological samples). Those who are willing to participate will sign the written informed consent. Participants would receive incentives for delaying his/her work by the interview. Incentives are gifts that are worth no more than $¥ 30$. For all questions, every effort was taken to ensure the accuracy of responses: interviewers are extensively trained, and all training is standardised nationally. Detailed error checks and quality control were incorporated during the interview procedure. Participants were informed in advance the designated times when the research teams would visit, and the blood was collected after an overnight fast. Blood collectors are equipped with extensive experience in blood collection, familiar with and skilled in the use of anticoagulation vacuum blood collection tubes.

For the newly added participants and follow-up elderly, face-to-face interviews are performed to fill out the questionnaire for interviews to the surviving participants to collect their health status, behavioural and sociodemographic circumstances. Questions, such as self-rated health, life satisfaction and Mini-Mental State Examination (MMSE) tests on cognitive function, are answered by the interviewees only. Other questions are answered by the interviewees themselves, as much as possible. We conducted proxy interviews among the close family member for those too frail to administer questionnaires. Meanwhile, physical examination is performed, and blood and urine sample are also collected. For those who have died since the last follow-up, investigators would endeavour to interview their next of kin and complete the questionnaire addressed to a close family member of the deceased, in order to collect relevant information before the death of the participants.

The on-site chief coordinator will randomly select two questionnaires collected by each interviewer in the previous day for comprehensive review. On the other hand, the prefectural on-site chief coordinator (quality controller) also conducts a comprehensive review at the night of the visit or the next day. If problems are found, the investigators should conduct a household survey or an additional telephone survey. If the telephone survey is unavailable, additional on-site investigations must be conducted. Finally, CCDC and PCDC also carry out on-site supervision and quality inspection during the investigation to ensure the process and quality of investigations.

\section{Data collection}

Questionnaire design in HABCS is based on international standards and adapted to the Chinese cultural context and carefully validated by the pilot studies. ${ }^{20}$ Questions would gather information on risk factors for healthy ageing and mortality, which are extremely comprehensive and are able to reflect a broad range of characteristics and conditions of the Chinese elderly. Details of the questionnaire for the surviving participants are shown in table 3. Briefly, by using questionnaires, the HABCS 
Table 3 Data collection from questionnaire for interviews to the surviving participants in HABCS

\begin{tabular}{|c|c|c|}
\hline \multicolumn{3}{|c|}{ Questionnaire for interviews to the surviving participants in HABCS } \\
\hline Basic information & Life evaluation and personality & Mini-Mental State Examination \\
\hline $\begin{array}{l}\text { Sex } \\
\text { Ethnic group } \\
\text { Age } \\
\text { Place of Birth } \\
\text { Characteristics of Current Residence }\end{array}$ & $\begin{array}{l}\text { Life satisfaction and self-rated health } \\
\text { Personality }\end{array}$ & $\begin{array}{l}\text { Orientation } \\
\text { Registration } \\
\text { Attention and calculation } \\
\text { Recall } \\
\text { Language }\end{array}$ \\
\hline Life style & $\begin{array}{l}\text { Activities of daily living (ADL) and Instrumental } \\
\text { ADL (IADL) }\end{array}$ & Personal background \\
\hline $\begin{array}{l}\text { Diet } \\
\text { Smoke } \\
\text { 1. Tobacco-nicotine dependence } \\
\text { 2. Personal perception and knowledge of } \\
\quad \text { smoking-related cancer risk } \\
\text { 3. Passive smoke exposure } \\
\text { Alcohol } \\
\text { 1. Alcohol-lifetime abuse and dependence } \\
\text { Exercises } \\
\text { Physical activity } \\
\text { Other activities }\end{array}$ & $\begin{array}{l}\text { Limited in Activities } \\
\text { ADL } \\
\text { 1. Bathing } \\
\text { 2. Dressing } \\
\text { 3. Toilet } \\
\text { 4. Indoor transfer } \\
\text { 5. Continence } \\
\text { 6. Eating } \\
\text { Characteristics of caregiver } \\
\text { IADL } \\
\text { 1. Cleaning } \\
\text { 2. Managing money } \\
\text { 3. Moving within the community } \\
\text { 4. Preparing meals } \\
\text { 5. Shopping } \\
\text { 6. Taking prescribed medications } \\
\text { 7. Using the telephone }\end{array}$ & $\begin{array}{l}\text { Education } \\
\text { Primary occupation before age } 60 \\
\text { Retirement age } \\
\text { Pension for retirement } \\
\text { Public old age insurance } \\
\text { Financial support } \\
\text { Economic status } \\
\text { Current marital status } \\
\text { Medical service } \\
\text { Social security and commercialised insurances } \\
\text { Childhood status } \\
\text { Mother's information } \\
\text { Father's information } \\
\text { Birth order } \\
\text { Biological brothers' and sisters' information } \\
\text { Children's information } \\
\text { Money gotten/given } \\
\text { Social services in the living community } \\
\text { Preference of living arrangement }\end{array}$ \\
\hline
\end{tabular}

Objective examination and illnesses attention

Sleep quality
Teeth status
Oral hygiene of personal care
Toothache and orofacial pain
Hand dominance
Standing height or self-reported height
Waist circumference
Hearing loss
Physical illnesses

HABCS, Healthy Aging and Biomarkers Cohort Study.

collects extensive information such as family structure, living arrangements, self-rated health, self-evaluation on life satisfaction, chronic disease, medical care, social activities, diet, smoking and alcohol drinking, psychological characteristics, economic resources, caregiver and family support, nutrition and some health-related conditions in early life. ${ }^{3}$ Activities of daily living (ADL) and cognitive function confirmed by the MMSE are also evaluated. $^{22} 23$ We documented the date of death from the family members of the deceased or local doctors. As for those who have died before the next wave, investigators will interview one of their next of kin. The collected information before dying includes date/cause of death, chronic diseases, ADL, number of hospitalisations or incidents of being bedridden from the last interview to death, and whether the participants have been able to obtain adequate medical treatment when suffering from disease. We also collect information on socioeconomic and demographic characteristics, such as marital status, family structure, caregivers, financial situation and living arrangement before death. In addition to administering structured questionnaires, physical examination is conducted, including blood pressure, heart rate, rhythm, height, weight, waist circumference, hip circumference, the ability to move physically, the ability to pick up ground books, number of steps needed to rotate one circle, respiratory function, grip force, nerves, hearing, chest, abdominal and language. Meanwhile, fasting blood samples as well as urine for biomarker measurements are collected. Blood samples were collected after an overnight fast (more than 12 hours). For all the interviewees, we collect $7 \mathrm{~mL}$ venous blood sample $(5+2 \mathrm{~mL})$ in total with two heparin anticoagulation blood collection tubes. One of the tubes with $5 \mathrm{~mL}$ blood sample is centrifuged to obtain plasma and blood cells, after which 1.0-1.2 mL of plasma is separately stored into two freezing tubes, whereas white blood cells layer is absorbed and stored in the other tube. The remaining $2 \mathrm{~mL}$ blood sample is stored into two freezing tube, with $1 \mathrm{~mL}$ blood for each for further analysing. Additionally, $50 \mathrm{~mL}$ urine sample is collected after an overnight fast (more than 12 hours). Specifically, $2 \mathrm{~mL}$ urine sample is stored into a freezing tube, while the remaining sample is stored into the other freezing tubes, with each tube storing $4.5 \mathrm{~mL}$ urine sample, resulting in 


\begin{tabular}{|c|c|}
\hline Biological samples & Specific biological indicators tested \\
\hline $\begin{array}{l}\text { Blood routine } \\
\text { examination }\end{array}$ & Blood platelet, haemoglobin, red blood cell, white blood cell. \\
\hline $\begin{array}{l}\text { Urine routine } \\
\text { examination }\end{array}$ & $\begin{array}{l}\text { Bilirubin, creatinine, erythrocyte, glucose, ketone body, leucocyte, microalbuminuria, nitrite, occult } \\
\text { blood, specific gravity, urine protein, urobilinogen, } \mathrm{pH} \text {. }\end{array}$ \\
\hline Blood biochemistry & $\begin{array}{l}\text { Fasting blood glucose, glycated albumin, high-density lipoprotein cholesterol, high-sensitivity C } \\
\text { reactive protein, low-density lipoprotein cholesterol, malondialdehyde, plasma albumin, serum } \\
\text { creatinine, superoxide dismutase, total cholesterol, triglyceride, uric acid, urea nitrogen, 25-hydroxy } \\
\text { vitamin D. }\end{array}$ \\
\hline
\end{tabular}

HABCS, Healthy Aging and Biomarkers Cohort Study.

nine tubes in total. Finally, the remaining urine sample is used for on-site creatinine detection. During on-site investigations, blood samples were centrifuged within 1 hour after blood collection for the separation of plasma from blood cell. Heparin anticoagulant blood samples were centrifuged at $3000 \mathrm{rpm}$ for $10 \mathrm{~min}$ at $18^{\circ} \mathrm{C}-25^{\circ} \mathrm{C}$. Then all plasma, white blood cells, whole blood and urine samples are stored at $-80^{\circ} \mathrm{C}$ in the county CDC. And then the sample were transported at $-20^{\circ} \mathrm{C}$ with transport cases provided by CCDC by specially assigned persons to designated testing units. After the plasma versus blood separation, natural sinking method was used to isolate white blood cells from red blood cells. We have added this information to the revised manuscript. A variety of biological indicators are analysed (table 4). All laboratory analyses are conducted by the central clinical laboratory at Capital Medical University in Beijing. Laboratory quality control material was run at the beginning of each shift. Levey-Jennings chart was plotted on to give a visual indication whether a laboratory test is working well. A mark is made indicating how far away the actual result was from the mean (which is the expected value for the control). Lines run across the graph at the mean, as well as 1, 2 and $3 \mathrm{SD}$ to either side of the mean. This makes it easy to see how far off the result was. The freeze thaw only occurred once from the collection of biological samples to the determination of biomarkers.

\section{Patient and public involvement}

None of the participants was involved in the questionnaire design, biological measurements or outcome measures; they were likewise not involved in the design, recruitment and implementation of the study. Furthermore, all participants or their relatives were informed of the use of the data for research in this study. There were no plans to disseminate the study results to participants.

\section{FINDINGS TO DATE}

As HABCS was established to investigate the determinants of healthy ageing. The biomarkers we focused on are either the confounding factors or the risk factors for adverse health outcomes in the older adults. In fact, recent publications of this cohort have reported links between biomarkers and mortality or cognitive impairment. Details of the findings are shown as follows:

\section{Lipid and cognitive function}

We have shown that higher baseline total cholesterol (TC) and low-density lipoprotein cholesterol (LDL-C) concentrations were associated with greater cognitive decline. ${ }^{24}$ Compared with the lowest quartiles, the corresponding adjusted mean differences in cognitive decline rate for TC and LDL-C were 0.28 (95\% CI -0.54 to -0.02; $\mathrm{p}$ for trend $=0.005)$ and $0.42(95 \% \mathrm{CI}-0.69$ to $0.16 ; \mathrm{p}$ for trend=0.006), respectively. Further, the associations between all lipids and cognitive decline appeared to be more pronounced among the centenarians, in comparison to other age groups. It should be cautious that these findings were not accounted for competing risks and potential reverse causation should also be taken into account due to the short follow-up period.

\section{Lipid and all-cause mortality}

HABCS has also observed that higher LDL-C level was associated with lower risk of all-cause mortality. ${ }^{25}$ Our results indicated that each $1 \mathrm{mmol} / \mathrm{L}$ increase of LDL-C concentration resulted in a $19 \%$ decrease in 3-year all-cause mortality (hazard ratio (HR) $0.81,95 \%$ CI 0.71 to 0.92 ). The adjusted HR for abnormally higher LDL-C concentration $(\geq 3.37 \mathrm{mmol} / \mathrm{L})$ was $0.60(95 \%$ CI 0.37 to 0.95$)$ compared with the low LDL-C concentration. This is the first study to investigate this association in a non-Western population. However, potential reverse causation might have affected the associations found.

\section{Vitamin D levels and cognitive function}

We have examined the cross-sectional association between quartiles of plasma vitamin D level and cognitive impairment (MMSE score $<18$ ) by using logistic regressions. ${ }^{26}$ Adjustments were made for demographics, diseases and various biomarkers. After adjusting for various covariates, there was a reverse association between plasma vitamin $\mathrm{D}$ and cognitive impairment. The multivariable adjusted odds ratio (OR) for lowest versus highest plasma vitamin D quartiles was 2.15 (95\% CI 1.05 to 4.41) for cognitive impairment, and the multivariable OR associated with 1 SD decrement of plasma vitamin D was 1.32 (95\% CI 1.00 
to 1.74) for cognitive impairment. Subgroup analyses have indicated that these associations did not vary significantly between gender and different age groups (all $\mathrm{p}$ for interaction $>0.05$ ). This study used the largest sample size of oldest old in Asia to explore relationship between plasma vitamin $\mathrm{D}$ and cognitive impairment.

Meanwhile, we have also investigated prospective associations between vitamin $\mathrm{D}$ levels at baseline and risk of subsequent cognitive decline and impairment. ${ }^{19}$ Participants with lower plasma vitamin D levels had a higher risk of a decrease of $\geq 3$ MMSE points over the 2-year follow-up in comparison to those with higher levels. The fully adjusted OR associated with $1 \mathrm{SD}$ decrement of plasma vitamin D levels was 1.35 (95\% CI 1.10 to 1.66) for cognitive decline. These results were not gender specific. Given the short follow-up period and high mortality rate, the reverse causation among this study population and the absence of accounting for competing risks from morality restricts interpretation of this results.

\section{Superoxide dismutase activity and cognitive function}

Researchers have also examined the association between plasma superoxide dismutase (SOD) activity and cognitive decline in HABCS. ${ }^{27}$ Participants in the highest quartile of SOD activity had an increased risk of cognitive decline in comparison to those in the lowest quartile (relative risk (RR) $1.32,95 \%$ CI 1.00 to $1.74, \mathrm{p}=0.051$ ).Using cut-off points determined by $\mathrm{X}^{2}$ automatic interaction detector analysis, the multivariable (RRs; 95\% CI) for the lowest category, second highest and the highest versus the third highest category of SOD activity were 0.56 (0.34 to 0.92 ), 1.26 (1.03 to 1.54 ) and 0.96 (0.70 to 1.31$)$, respectively. This study indicated that a higher SOD activity was associated with elevated risk of cognitive decline among Chinese older adults. These results were generally consistent after accounting for competing risks from mortality. However, reverse causation should be taken into account giving the relatively short follow-up period.

\section{STRENGTHS AND LIMITATIONS}

As for strengths, we select a representative sample of centenarians, nonagenarians and octogenarians from eight longevity areas through a special design that resembles a case-control design, which may offer a unique data set of oldest old in a non-Western country. Further, the HABCS provides data on under-represented age groups (eg, centenarians), for whom comparisons often lack statistical power due to limited sample sizes. Also, all the interviewees are interviewed face to face at home, thus limiting the under-representation of frailer participants. Another key strength is that the field and research teams have extensive experience implementing fieldwork, and a variety of healthcare facilities have participated in the project, with the CCDC and PCDC providing project review and validation as well as technical guidance. This multicollaboration system would insure the quality of our research. In addition, most of the variables were repeatedly collected through multiple waves, which would facilitate the tracing of trajectories of healthy ageing. Besides, all laboratory analyses were centrally conducted by a central clinical laboratory at China Capital Medical University, which is equipped with requisite skills and facilities, thereby eliminating interlaboratory assay. More importantly, we collect comprehensive data by including a broad range of variables especially biological indicators, all of which will allow meaningful adjustment of various confounders when dissecting the complex inter-relationships of factors and identifying modifiable risk factors and biomarkers for healthy ageing. Last but not least, the interview refusal rate among the Chinese oldest old was very low: about $2 \%$ among those who were not too sick to participate with proxy assistance.

Several limitations should be taken into consideration: (1) our study mainly focuses on Chinese oldest old, so our findings may not be applicable to other ethnic or age groups. (2) The areas (including Rudong County) we selected have higher densities of centenarians and higher life expectancies, this may partly due to the special environmental influences plus socioeconomic factors. Thus, HABCS may not be representative of the general population in China. (3) The relative high rate of lost to follow-up due to the old age and low education status of our participants may bring bias. (4) Numbers of each matched age group is not as exact as we initially designed because matching eligible oldest old is difficult during field work. (5) One would argue that attrition from wave to wave is inherent in most longitudinal studies of elderly, which would result in positive selection effects. However, we try to re-interview all of the elderly who are lost to follow-up in each wave and replace for the deceased elderly, through which the sample size would be relatively stable over time.

Acknowledgements We thank the participants who contributed to this cohort.

Contributors The study protocol was designed by XS, YL and CM drafted the manuscript. ZY, FL and XW performed the data quality control and the statistical analysis. All authors participated in the study design, revised the article and approved the final version.

Funding The HABCS is jointly supported by National Natural Sciences Foundation of China (grant number 71233001, 71490732 to ZY and 81273160, 81573247 to XS.), the US National Institute of Aging (grant number 2P01AG031719 to ZY) and United Nations Fund for Population Activities, and a Claude D. Pepper Older Americans Independence Centers grant (5P30AG028716 from NIA to VBK).

Map disclaimer The depiction of boundaries on the map(s) in this article do not imply the expression of any opinion whatsoever on the part of BMJ (or any member of its group) concerning the legal status of any country, territory, jurisdiction or area or of its authorities. The map(s) are provided without any warranty of any kind, either express or implied.

Competing interests None declared.

Patient consent for publication Not required.

Ethics approval The study was approved by the biomedical ethics committee of Peking University and Duke University.

Provenance and peer review Not commissioned; externally peer reviewed.

Data availability statement The data of HABCS are publicly available. More details about HABCS are available on the website: (http://www.habcs.cn/). We welcome joint analyses of the cohort data. Any research group can submit a research proposal providing information on background, research questions, 
methods, timetable and budget as well as authorship for new collaborations. Research proposals will be reviewed by scientific committee.

Open access This is an open access article distributed in accordance with the Creative Commons Attribution Non Commercial (CC BY-NC 4.0) license, which permits others to distribute, remix, adapt, build upon this work non-commercially, and license their derivative works on different terms, provided the original work is properly cited, appropriate credit is given, any changes made indicated, and the use is non-commercial. See: http://creativecommons.org/licenses/by-nc/4.0/.

\section{REFERENCES}

1 Gómez-Olivé FX, Montana L, Wagner RG, et al. Cohort profile: health and ageing in Africa: a longitudinal study of an indepth community in South Africa (HAALSI). Int J Epidemiol 201810.1093/ije/dyx247. [Epub ahead of print: 06 Jan 2018].

2 Collerton J, Davies K, Jagger C, et al. Health and disease in 85 year olds: baseline findings from the Newcastle $85+$ cohort study. BMJ 2009;339:b4904.

3 Zeng Y, Feng Q, Hesketh T, et al. Survival, disabilities in activities of daily living, and physical and cognitive functioning among the oldestold in China: a cohort study. The Lancet 2017;389:1619-29.

4 Zhou Z, Wang P, Fang Y. Social engagement and its change are associated with dementia risk among Chinese older adults: a longitudinal study. Sci Rep 2018;8:1551.

5 Pham-Kanter G, Goldman N. Do sons reduce parental mortality? J Epidemiol Community Health 2012;66:710-5.

6 Feng Q, Zhu H, Zhen Z, et al. Self-Rated health, Interviewer-Rated health, and their predictive powers on mortality in old age. $J$ Gerontol B Psychol Sci Soc Sci 2016;71:538-50.

7 Yang L, Konttinen H, Martikainen P, et al. Socioeconomic status and physical functioning: a longitudinal study of older Chinese people. $J$ Gerontol B Psychol Sci Soc Sci 2018;73:1315-29.

$8 \mathrm{Ng} \mathrm{ST}$, Tey NP, Asadullah MN. What matters for life satisfaction among the oldest-old? Evidence from China. PLoS One 2017;12:e171799.

9 Liu G, Dupre ME, Gu D, et al. Psychological well-being of the institutionalized and community-residing oldest old in China: the role of children. Soc Sci Med 2012;75:1874-82.

10 Shen K, Zeng Y. Direct and indirect effects of childhood conditions on survival and health among male and female elderly in China. Soc Sci Med 2014;119:207-14.

11 Luo Y, Zhang Z, Gu D. Education and mortality among older adults in China. Soc Sci Med 2015;127:134-42.

$12 \mathrm{Li} \mathrm{T}$, Zhang Y. Social network types and the health of older adults: exploring reciprocal associations. Soc Sci Med 2015;130:59-68.
$13 \mathrm{Xu} \mathrm{H}$, Dupre ME, Gu D, et al. The impact of residential status on cognitive decline among older adults in China: results from a longitudinal study. BMC Geriatr 2017;17.

14 Gu D, Yang F, Sautter J. Socioeconomic status as a moderator between frailty and mortality at old ages. Bmc Geriatr 2016;16.

15 Yang L, Martikainen P, Silventoinen K. Effects of individual, spousal, and offspring socioeconomic status on mortality among elderly people in China. J Epidemiol 2016;26:602-9.

16 Cheng L, Liu H, Zhang Y, et al. The impact of health insurance on health outcomes and spending of the elderly: evidence from China's new cooperative medical scheme. Health Econ 2015;24:672-91.

17 Cai J, Zhao H, Coyte P. Socioeconomic differences and trends in the place of death among elderly people in China. Int J Environ Res Public Health 2017;14:1210.

18 Wang D, Zheng J, Kurosawa M, et al. Changes in activities of daily living (ADL) among elderly Chinese by marital status, living arrangement, and availability of healthcare over a 3-year period. Environ Health Prev Med 2009;14:128-41.

19 Matchar DB, Chei C-L, Yin Z-X, et al. Vitamin D levels and the risk of cognitive decline in Chinese elderly people: the Chinese longitudinal healthy longevity survey. J Gerontol A Biol Sci Med Sci 2016;71:1363-8.

$20 \mathrm{Yi}$, Jr, Poston DL, Vlosky DA, et al. Healthy longevity in China. Durham, North Carolina, USA: Springer, 2008.

21 Lv Y-B, Gao X, Yin Z-X, et al. Revisiting the association of blood pressure with mortality in oldest old people in China: community based, longitudinal prospective study. BMJ 2018;361:k2158.

22 Lv Y-B, Yuan J-Q, Mao C, et al. Association of body mass index with disability in activities of daily living among Chinese adults 80 years of age or older. JAMA Netw Open 2018;1:e181915.

23 Yuan J-Q, Lv Y-B, Chen H-S, et al. Association between latelife blood pressure and the incidence of cognitive impairment: a community-based prospective cohort study. J Am Med Dir Assoc 2019;20:177-82.

$24 \mathrm{Ma} \mathrm{C}$, Yin Z, Zhu P, et al. Blood cholesterol in late-life and cognitive decline: a longitudinal study of the Chinese elderly. Mol Neurodegener 2017;12.

25 Lv Y-B, Yin Z-X, Chei C-L, et al. Low-Density lipoprotein cholesterol was inversely associated with 3-year all-cause mortality among Chinese oldest old: data from the Chinese longitudinal healthy longevity survey. Atherosclerosis 2015;239:137-42.

26 Chei C-L, Raman P, Yin Z-X, et al. Vitamin D levels and cognition in elderly adults in China. $J$ Am Geriatr Soc 2014;62:2125-9.

27 Sun D, Sun X, Xu Y, et al. Superoxide dismutase activity and risk of cognitive decline in older adults: findings from the Chinese longitudinal healthy longevity survey. Exp Gerontol 2019;118:72-7. 